\title{
Análisis Morfológico del Cóndilo Mandibular por Tomografía Computarizada de Haz Cónico en Relación al Sexo en Población Mexicana
}

\author{
Morphological Analysis of the Mandibular Condyle by Cone Beam \\ Computed Tomography in Relation to Sex in a Mexican Population
}

José Carlos López Ramírez; José Obed García Cortes; Wulfrano Sánchez Meraz; Ricardo Oliva Rodríguez; Francisco Javier Gutiérrez Cantú \& Jairo Mariel Cárdenas

LÓPEZ, R. J. C.; GARCÍA, C. J. O.; SÁNCHEZ, M. W.; OLIVA, R. W.; GUTIÉRREZ, C. F. J. \& CÁRDENAS, J. M. Análisis morfológico del cóndilo mandibular por tomografía computarizada de haz cónico en relación al sexo en población mexicana. Int. J. Morphol., 39(5):1420-1428, 2021.

RESUMEN: La articulación temporomandibular es una estructura doble y simétrica, con una compleja gama de movimientos. La morfología condilar puede variar dependiendo al sexo, edad, entre otros factores. Se analizaron 100 tomografías computarizadas de haz cónico (50 hombres y 50 mujeres) divididos en 4 grupos. Se identificó diferencias significativas entre las formas aplanadas vs ovaladas del cóndilo y a su vez se encontró diferencia entre los lados derechos vs izquierdo entre hombres y mujeres. La tomografía computada de haz cónico es un método económico y accesible mediante la exposición a bajas dosis de radiación se obtienen imágenes de alta resolución de los tejidos duros de la ATM. El análisis de la tomografía computarizada de haz cónico tiene una precisión aceptable para el diagnóstico de anomalías óseas de la ATM, componentes articulares, integridad del hueso cortical y anomalías óseas subcorticales.

PALABRAS CLAVE: Articulación temporomandibular; Tomografía computarizada de haz cónico; Desórdenes temporomandibulares.

\section{INTRODUCCIÓN}

La articulación emporomandibular (ATM) es una estructura doble y simétrica, una sinovial bicondílea con una compleja gama de movimientos que incluye 6 tipos: apertura, cierre, protrusión, retrusión y lateralización a derecha e izquierda. (Ros et al., 2008). La ATM es fundamental para las funciones vitales del individuo en funciones como la masticación, deglución y habla que permiten que el complejo temporomandibular ejecute los movimientos de deslizamiento, apertura y cierre mandibular. (Chavarro et al., 2016). La capacidad funcional se basa en una compleja anatomía constituida en la parte superior por la fosa glenoidea y la eminencia articular, ambas estructuras del hueso temporal, y en la parte inferior por la cabeza del cóndilo mandibular (Ros et al.). El proceso condilar presenta el cóndilo mandibular que es una eminencia ovoidea cuyo eje mayor está dirigido hacia atrás y adentro unida a la rama mandibular mediante un cuello más estrecho que es más fino en su parte antero medial donde se inserta el músculo pterigoideo lateral. El proceso condilar está formado por el cóndilo mandibular (cabeza mandibular) y cuello mandibular además de la fosa pterigoidea. La cabeza mandibular es convexa en todos los sentidos, especialmente en sentido anteroposterior. Su superficie superior es la que articula con el temporal. Su eje longitudinal es perpendicular a la rama mandibular. (Farfan et al.,2020). Los autores Oberg, Carlsson y Falers clasificaron en diferentes grupos los cóndilos mandibulares y los tubérculos articulares de acuerdo con su apariencia general en los siguientes grupos: 1 . Redondeado 2. plano. 3. En forma de cresta (V invertida), 4. Otras formas. El contorno horizontal del cóndilo desde una vista superior se clasifica en: 1 . Oblongo,2. Redondeado, 3. forma de pera, 4. Cónico y 5. Otras formas (Torsten et al., 1971). Se ha discutido sobre anatomíay la relación funcional entre el cóndilo mandibular y la cavidad articular, incluso durante el desarrollo, se considera genéricamente la fosa con una forma redondeada o simplemente una depresión en la parte escamosa del hueso temporal donde se articula el cóndilo mandibular. Descripciones y clasificaciones de la forma de 
LÓPEZ, R. J. C.; GARCÍA, C. J. O.; SÁNCHEZ, M. W.; OLIVA, R. W.; GUTIÉRREZ, C. F. J. \& CÁRDENAS, J. M. Análisis morfológico del cóndilo mandibular por tomografía computarizada de haz cónico en relación al sexo en población mexicana. Int. J. Morphol., 39(5):1420-1428, 2021.

la fosa son raras en la literatura. Se ha examinado el cóndilo mandibular y la morfología de la fosa mandibular en imágenes de tomografía computarizada (TC) obtenido de pacientes con desórdenes temporomandibulares y pacientes sanos, que describe cuatro formas de fosa mandibular. También se ha observado la forma y ubicación de la fosa mandibular y su relación con la asimetría mandibulofacial mediante radiografías de cráneo seco. Este no ha sido el caso del cóndilo mandibular, donde la clasificación de formas ha sido presentada por Ribeiro et al., 2015. Matsumoto et al. (2013) investigaron las características morfológicas del cóndilo mandibular y la fosa mandibular con la vista anteroposterior y mostraron las características de la morfología coronal. En la evaluación tridimensional de pacientes con enfermedad de ATM (como con tomografía computarizada o resonancia magnética), se pueden utilizar imágenes seccionales coronales. La resonancia magnética coronal de la ATM también se puede utilizar en la evaluación de la posición del disco articular.

Existen muchas lesiones, enfermedades inflamatorias y degenerativas, trastornos posturales, traumas y otras anormalidades que comprometen el equilibrio de los componentes de la articulación temporomandibularlo que resulta en su trastorno o disfunción (Marques et al., 2010). Las causas originales de los trastornos de ATM son generalmente complejas. Algunos clínicos consideran la disfunción del músculo pterigoideo lateral como un posible causante factor en el trastorno interno de la ATM. Sin embargo, algunos los pacientes pueden sufrir episodios recurrentes de ATM debido a un exceso de contracción de los ligamentos superiores. (Omami \& Lurie, 2012).

Un examen radiográfico es parte de la rutina de evaluación clínica para las condiciones de disfunción de la ATM y el objetivo principal es verificar los cambios óseos degenerativos en las estructuras articulares y la amplitud de la excursión condilar (Dos Anjos Pontual et al., 2012). La resonancia magnética es capaz de proporcionar información sobre la morfología y posición del disco a través de alta resolución de tejidos blandos sin exponer al paciente a radiación ionizante. (Omami \& Lurie). Así mismo con la TC es posible analizar el límite del cóndilo mandibular, su posición en la fosa mandibular y la afectación del hueso cortical en relación con las lesiones óseas. Varias alteraciones óseas que se producen en la ATM, como erosiones y osteofitos en el cóndilo mandibular, son difíciles de detectar con la radiografía convencional y, por lo tanto, requieren el uso de la TC (Honey et al., 2007). Con la llegada de la Tomografía Computarizada de haz cónico (CBCT) estas barreras se han superado. Hoy en día, las unidades CBCT están ubicadas en escuelas de odontología, laboratorios radiográficos dentales y consultorios privados, y han proporcionado un mayor acceso a la tecnología CT. Ofrece varias ventajas sobre la tomografía computarizada médica tales como la dosis de radiación de los exámenes suele ser mucho más baja que la de las unidades de TC y la resolución espacial de las imágenes suele ser más alta que la de los protocolos de imágenes de resonancias magnéticas. La eficacia diagnóstica es tan buena como la TC convencional y es superior a la de la radiografía panorámica y la tomografía lineal. Dada la menor dosis de radiación y la creciente disponibilidad se está convirtiendo en la modalidad de elección para la evaluación de los componentes óseos de la ATM (Barghan et al., 2012).

Esta herramienta de diagnóstico por imágenes brinda a los médicos nuevas oportunidades y responsabilidades para un mejor diagnóstico de las estructuras que se muestran en las imágenes CBCT incluida la ATM. Varios estudios recientes sugirieron que es una herramienta prometedora para diagnosticar afecciones óseas de la ATM (Iskanderani et al., 2020). El objetivo de la presente investigación fue determinar las diferencias morfológicas del cóndilo mandibular por tomografía computarizada de haz cónico en relación con el sexo de la muestra integrada por individuos de población mexicana.

\section{MATERIAL Y METODO}

Se analizaron 100 tomografías computarizadas de haz cónico (50 hombres y 50 mujeres) divididos en 4 grupos (cóndilo mandibular izquierdo hombres, cóndilo mandibular derecho hombres, cóndilo mandibular izquierdo mujeres, cóndilo mandibular derecho mujeres) con edades de 18 a 35 años que asistieron a consulta en la Maestría de Ciencias Odontológicas de la Universidad Autónoma de San Luis Potosí entre los años de 2017 y 2018. Se observó la morfología del cóndilo mandibular con las siguientes medidas: 1. Altura del cóndilo mandibular derecho, 2. Altura del cóndilo mandibular izquierdo, 3. Ancho vestíbulo-lingual derecho, 4. Ancho vestíbulo-lingual izquierdo, 5. Ancho frontal-posterior derecho, 6. Ancho frontal-posterior izquierdo. Se analizó la densidad ósea: 1 . altura apical, 2. altura media, 3. parte coronal, 4. anchura vestibular, 5. anchura media, 6 . anchura palatina.Se realizó un estudio transversal retrospectivo, a partir de tomografías y se analizaron las tomografías al azar entre los años 2017 y 2018, San Luis Potosí, S.L.P. con un software de Caresteam de Kodak utilizando una laptop ASUS vivobook X510U. Para el análisis estadístico se utilizó el software Minitab versión 19 en español. Se determino la estadística descriptiva y se analizó la normalidad de las variables con la prueba de ShapiroWilk, para determinar el tipo de prueba estadística a aplicar en el estudio y determinar la significancia estadística con intervalos de confianza al $95 \%$. 

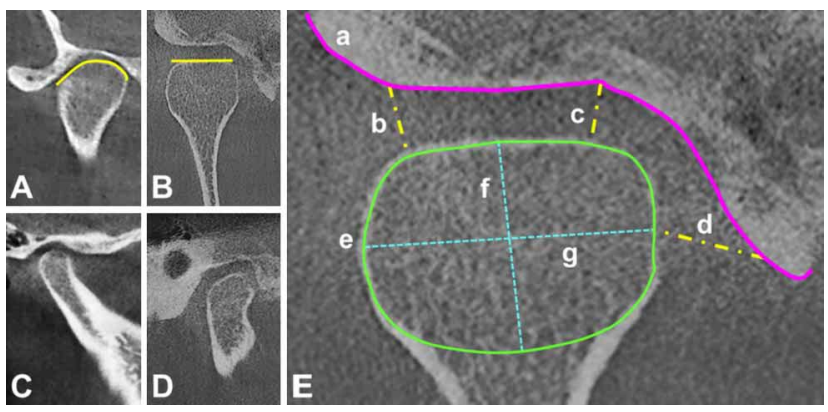

Fig. 1. Condilo Mandibular. Corte coronal: A. ovalado, B. Plano; Corte sagital: C. ovalado, D: Plano; E. Trazado de longitudes y estructuras: a. cavidad glenoidea, b. distancia de la pared posterior al cóndilo mandibular, c. distancia de la pared superior al cóndilo mandibular, d. distancia de la pared anterior al cóndilo mandibular, e. cóndilo mandibular, F. altura y g. ancho.

Tabla I. Estadística descriptiva del cóndilo mandibular izquierdo de hombres, en 50 tomografías computarizadas de haz cónico.

\begin{tabular}{|c|c|c|c|c|c|c|c|c|}
\hline $\begin{array}{l}\text { Forma del } \\
\text { cóndilo } \\
\text { mandibular }\end{array}$ & Variable & $\begin{array}{l}\text { Lado del } \\
\text { cóndilo } \\
\text { mandibular }\end{array}$ & Media & $\begin{array}{c}\text { Error de la } \\
\text { media }\end{array}$ & DS & Mínimo & Mediana & Máximo \\
\hline \multirow[t]{15}{*}{ Aplanada } & \multirow[t]{2}{*}{ Altura } & Derecho & 19.275 & 0.692 & 2.188 & 16.18 & 19.63 & 21.95 \\
\hline & & Izquierdo & 18.311 & 0.797 & 2.52 & 14.19 & 17.615 & 22.27 \\
\hline & \multirow[t]{2}{*}{ Ancho vestíbulo lingual } & Derecho & 14.901 & 0.955 & 3.021 & 10.75 & 15.305 & 21.24 \\
\hline & & Izquierdo & 15.122 & 0.655 & 2.071 & 12.46 & 15.22 & 19.17 \\
\hline & \multirow[t]{2}{*}{ Ancho frontal posterior } & Derecho & 7.132 & 0.347 & 1.097 & 5.41 & 6.79 & 9.43 \\
\hline & & Izquierdo & 6.591 & 0.44 & 1.393 & 3.94 & 6.575 & 8.69 \\
\hline & \multirow[t]{3}{*}{ Densidad de la atura } & Apical & 749 & 132 & 416 & 235 & 667 & 16070 \\
\hline & & Media & 269.6 & 47.6 & 150.6 & 80 & 231 & 524 \\
\hline & & Coronal & 397.2 & 80.2 & 253.6 & 56 & 364.5 & 740 \\
\hline & \multirow[t]{3}{*}{ Densidad del ancho } & Vestibular & 359 & 74.5 & 235.6 & 105 & 323 & 742 \\
\hline & & Media & 210.5 & 33.5 & 106 & 97 & 184.5 & 372 \\
\hline & & Palatino & 430.4 & 79.5 & 251.3 & 183 & 336 & 1036 \\
\hline & \multirow[t]{3}{*}{ Densidad pared } & Anterior & 2.19 & 0.227 & 0.719 & 1.26 & 1.995 & 3.78 \\
\hline & & Posterior & 2.306 & 0.246 & 0.776 & 1.3 & 2.21 & 3.91 \\
\hline & & Superior & 3.082 & 0.202 & 0.64 & 2.31 & 3.075 & 4.19 \\
\hline \multirow[t]{15}{*}{ Ovalada } & \multirow[t]{2}{*}{ Altura } & Derecho & 22.848 & 0.607 & 1.918 & 18.89 & 22.71 & 25.86 \\
\hline & & Izquierdo & 22.467 & 0.831 & 2.629 & 17.53 & 22.27 & 26.94 \\
\hline & \multirow[t]{2}{*}{ Ancho vestíbulo lingual } & Derecho & 17.254 & 0.647 & 2.045 & 12.46 & 17.87 & 20.47 \\
\hline & & Izquierdo & 15.986 & 0.658 & 2.082 & 13.28 & 15.585 & 20.09 \\
\hline & \multirow[t]{2}{*}{ Ancho frontal posterior } & Derecho & 7.293 & 0.39 & 1.233 & 5.39 & 7.215 & 9.06 \\
\hline & & Izquierdo & 7.318 & 0.302 & 0.954 & 5.91 & 7.26 & 8.88 \\
\hline & \multirow[t]{3}{*}{ Densidad de la atura } & Apical & 622.6 & 81.2 & 256.7 & 248 & 642.5 & 949 \\
\hline & & Medio & 336.1 & 76.1 & 240.6 & 14 & 249.5 & 748 \\
\hline & & Coronal & 513.5 & 76.7 & 242.6 & 201 & 471 & 886 \\
\hline & \multirow[t]{3}{*}{ Densidad del ancho } & Vestibular & 384.3 & 90.1 & 285 & 96 & 287 & 1002 \\
\hline & & Medio & 306.8 & 61.5 & 194.6 & 36 & 329 & 625 \\
\hline & & Palatino & 444.2 & 65.5 & 207.2 & 160 & 389 & 897 \\
\hline & \multirow[t]{3}{*}{ Densidad pared } & Anterior & 1.959 & 0.324 & 1.025 & 1.08 & 1.325 & 3.61 \\
\hline & & Posterior & 2.119 & 0.258 & 0.815 & 1.13 & 2.02 & 3.55 \\
\hline & & Superior & 2.842 & 0.22 & 0.694 & 1.75 & 2.85 & 3.89 \\
\hline
\end{tabular}




\section{RESULTADOS}

Se determinó la estadística descriptiva para el lado izquierdo y derecho de hombres y mujeres, la cual incluye media, error de la media, desviación estándar, mediana y valores mínimos y máximos (Tablas I a IV).
Se compararon las formas aplanadas vs ovalada de cóndilo para cada variable entre los grupos de estudio y se identificó significancia estadística en el lado izquierdo de hombres en las siguientes variables: altura del lado derecho del cóndilo ( $\mathrm{p} \leq 0.0008)$ e izquierdo ( $\mathrm{p} \leq 0.0046)$, ancho vestibulolingual del lado derecho ( $\mathrm{p} \leq 0.0172)$; en el lado derecho de hombres: altura del lado derecho del cóndilo ( $\mathrm{p}$

Tabla II. Estadística descriptiva del cóndilo mandibular derecho de hombre en 50 tomografías computarizadas de haz cónico.

\begin{tabular}{|c|c|c|c|c|c|c|c|c|}
\hline $\begin{array}{l}\text { Forma del } \\
\text { cóndilo } \\
\text { mandibular }\end{array}$ & Variable & $\begin{array}{c}\text { Lado del } \\
\text { cóndilo } \\
\text { mandibular }\end{array}$ & Media & $\begin{array}{l}\text { Error de la } \\
\text { media }\end{array}$ & $\begin{array}{l}\text { Desviación } \\
\text { estándar }\end{array}$ & Mínimo & Mediana & Máximo \\
\hline \multirow[t]{15}{*}{ Aplanada } & Altura & Derecho & 19.828 & 0.969 & 3.064 & 16.18 & 19.63 & 25.86 \\
\hline & & Izquierdo & 19.37 & 1.17 & 3.7 & 14.19 & 19.02 & 26.94 \\
\hline & $\begin{array}{l}\text { Ancho vestíbulo } \\
\text { lingual }\end{array}$ & Derecho & 15.53 & 1.03 & 3.27 & 10.75 & 16.07 & 21.24 \\
\hline & & Izquierdo & 16.007 & 0.838 & 2.651 & 12.46 & 15.735 & 20.09 \\
\hline & $\begin{array}{l}\text { Ancho frontal } \\
\text { posterior }\end{array}$ & Derecho & 7.282 & 0.397 & 1.256 & 5.41 & 7.275 & 9.43 \\
\hline & & Izquierdo & 6.821 & 0.471 & 1.489 & 3.94 & 6.575 & 8.69 \\
\hline & $\begin{array}{l}\text { Densidad de la } \\
\text { atura }\end{array}$ & Apical & 614.4 & 98.8 & 312.4 & 235 & 640 & 1102 \\
\hline & & Media & 253.6 & 53 & 167.6 & 14 & 227 & 524 \\
\hline & & Coronal & 387.1 & 66.2 & 209.4 & 56 & 384 & 705 \\
\hline & $\begin{array}{l}\text { Densidad del } \\
\text { ancho }\end{array}$ & Vestibular & 290.9 & 57.6 & 182.2 & 105 & 287 & 742 \\
\hline & & Media & 167.5 & 30.9 & 97.7 & 36 & 137.5 & 349 \\
\hline & & Palatino & 403.3 & 51.3 & 162.3 & 183 & 370 & 685 \\
\hline & Densidad pared & Anterior & 2.241 & 0.25 & 0.79 & 1.26 & 1.965 & 3.78 \\
\hline & & Posterior & 2.38 & 0.252 & 0.797 & 1.3 & 2.26 & 3.91 \\
\hline & & Superior & 2.804 & 0.193 & 0.611 & 1.74 & 2.63 & 3.72 \\
\hline \multirow[t]{15}{*}{ Ovalada } & Altura & Derecho & 22.662 & 0.538 & 1.701 & 18.89 & 22.965 & 24.61 \\
\hline & & Izquierdo & 21.704 & 0.849 & 2.686 & 17.27 & 22.27 & 25.34 \\
\hline & $\begin{array}{l}\text { Ancho vestíbulo } \\
\text { lingual }\end{array}$ & Derecho & 17.283 & 0.552 & 1.745 & 13.98 & 17.87 & 20.47 \\
\hline & & Izquierdo & 15.874 & 0.517 & 1.636 & 13.28 & 16.015 & 18.03 \\
\hline & $\begin{array}{l}\text { Ancho frontal } \\
\text { posterior }\end{array}$ & Derecho & 7.014 & 0.347 & 1.097 & 5.39 & 7.06 & 9.06 \\
\hline & & Izquierdo & 7.061 & 0.305 & 0.963 & 5.79 & 7.155 & 8.88 \\
\hline & $\begin{array}{l}\text { Densidad de la } \\
\text { atura }\end{array}$ & Apical & 686 & 127 & 402 & 307 & 604 & 1607 \\
\hline & & Medio & 395.7 & 65.4 & 206.8 & 142 & 392.5 & 748 \\
\hline & & Coronal & 587.2 & 67.5 & 213.4 & 201 & 644.5 & 839 \\
\hline & $\begin{array}{l}\text { Densidad del } \\
\text { ancho }\end{array}$ & Vestibular & 477.9 & 92.3 & 291.7 & 96 & 488 & 1002 \\
\hline & & Medio & 378.8 & 60.1 & 190.1 & 53 & 414 & 625 \\
\hline & & Palatino & 506 & 78.5 & 248.3 & 160 & 527 & 1036 \\
\hline & Densidad pared & Anterior & 1.724 & 0.239 & 0.757 & 1.08 & 1.39 & 3.61 \\
\hline & & Posterior & 1.964 & 0.162 & 0.512 & 1.13 & 1.97 & 2.91 \\
\hline & & Superior & 2.969 & 0.194 & 0.612 & 1.75 & 2.92 & 3.89 \\
\hline
\end{tabular}


$\leq 0.0172$ ), densidad de la altura del lado coronal ( $\mathrm{p} \leq 0.0376$ ). En el lado derecho de mujeres: densidad del ancho del lado medio ( $\mathrm{p} \leq 0.0121)$ y densidad pared anterior $(\mathrm{p} \leq 0.0375)$, no así en los demás grupos de estudio (Tabla V).

Se compararon las formas aplanadas y ovaladas para cada variable entre los grupos de estudio y se identificó significancia estadística en forma aplanada de hombres en las siguientes variables: en forma ovalada de hombres: densidad del ancho del lado medio ( $\mathrm{p} \leq 0.0403$ ); no así en los demás grupos de estudio (Tabla VI).

Se compararon las formas aplanada vs ovalada de cóndilo para cada variable entre los grupos de estudio y se

Tabla III. Estadística descriptiva del cóndilo mandibular izquierdo de mujeres en 50 tomografías computarizadas de haz cónico.

\begin{tabular}{|c|c|c|c|c|c|c|c|c|}
\hline $\begin{array}{l}\text { Forma del } \\
\text { cóndilo } \\
\text { mandibular }\end{array}$ & Variable & $\begin{array}{l}\text { Lado del } \\
\text { cóndilo } \\
\text { mandibular }\end{array}$ & Media & $\begin{array}{c}\text { Errorde la } \\
\text { media }\end{array}$ & $\begin{array}{l}\text { Desviación } \\
\text { estándar }\end{array}$ & Mínimo & Mediana & Máximo \\
\hline \multirow[t]{15}{*}{ Aplanada } & Altura & Derecho & 16.879 & 0.658 & 2.079 & 13.74 & 16.775 & 21.2 \\
\hline & & Izquierdo & 15.953 & 0.664 & 2.099 & 13.11 & 15.99 & 19.22 \\
\hline & $\begin{array}{c}\text { Ancho vestíbulo } \\
\text { lingual }\end{array}$ & Derecho & 14.206 & 0.804 & 2.544 & 10.14 & 14.215 & 18.72 \\
\hline & & Izquierdo & 14.451 & 0.728 & 2.303 & 11 & 14.31 & 17.88 \\
\hline & $\begin{array}{l}\text { Ancho frontal } \\
\text { posterior }\end{array}$ & Derecho & 7.118 & 0.399 & 1.262 & 5.06 & 7.34 & 9.09 \\
\hline & & Izquierdo & 7.181 & 0.436 & 1.377 & 4.98 & 7.31 & 8.98 \\
\hline & $\begin{array}{c}\text { Densidad de la } \\
\text { atura }\end{array}$ & Apical & 746 & 149 & 471 & 152 & 799 & 1549 \\
\hline & & Media & 398.5 & 55.5 & 175.6 & 90 & 381 & 669 \\
\hline & & Coronal & 546.6 & 70.8 & 224 & 264 & 602 & 1004 \\
\hline & $\begin{array}{l}\text { Densidad del } \\
\text { ancho }\end{array}$ & Vestibular & 567 & 78.9 & 249.5 & 267 & 548 & 1073 \\
\hline & & Media & 345.9 & 38.9 & 122.9 & 185 & 358 & 495 \\
\hline & & Palatino & 543.5 & 70.5 & 222.9 & 169 & 544 & 855 \\
\hline & Densidad pared & Anterior & 1.805 & 0.144 & 0.457 & 1.14 & 1.775 & 2.76 \\
\hline & & Posterior & 2.394 & 0.278 & 0.879 & 1.62 & 1.945 & 4.2 \\
\hline & & Superior & 2.575 & 0.317 & 1.002 & 1.62 & 2.4 & 5.17 \\
\hline \multirow[t]{15}{*}{ Ovalada } & Altura & Derecho & 17.497 & 0.733 & 2.318 & 14.47 & 17.56 & 21.91 \\
\hline & & Izquierdo & 17.292 & 0.693 & 2.193 & 14.91 & 16.935 & 20.84 \\
\hline & $\begin{array}{c}\text { Ancho vestíbulo } \\
\text { lingual }\end{array}$ & Derecho & 14.082 & 0.792 & 2.503 & 10.65 & 14.665 & 18.39 \\
\hline & & Izquierdo & 12.917 & 0.828 & 2.617 & 9.8 & 11.83 & 16.71 \\
\hline & $\begin{array}{c}\text { Ancho frontal } \\
\text { posterior }\end{array}$ & Derecho & 7.481 & 0.315 & 0.997 & 5.53 & 7.555 & 9.09 \\
\hline & & Izquierdo & 7.488 & 0.365 & 1.156 & 5.46 & 7.56 & 9.2 \\
\hline & $\begin{array}{c}\text { Densidad de la } \\
\text { atura }\end{array}$ & Apical & 897 & 138 & 436 & 326 & 683 & 1585 \\
\hline & & Medio & 377.2 & 77 & 243.4 & 130 & 307 & 865 \\
\hline & & Coronal & 681.1 & 82.9 & 262.2 & 359 & 660 & 1137 \\
\hline & $\begin{array}{l}\text { Densidad del } \\
\text { ancho }\end{array}$ & Vestibular & 626.6 & 94.5 & 298.8 & 185 & 611 & 1026 \\
\hline & & Medio & 339.7 & 46.3 & 146.4 & 79 & 326.5 & 562 \\
\hline & & Palatino & 528.7 & 68.3 & 216.1 & 263 & 549 & 775 \\
\hline & Densidad pared & Anterior & 2.198 & 0.16 & 0.507 & 1.39 & 2.135 & 3.04 \\
\hline & & Posterior & 2.526 & 0.377 & 1.194 & 1.78 & 2.115 & 5.82 \\
\hline & & Superior & 2.751 & 0.396 & 1.252 & 1.75 & 2.65 & 6.07 \\
\hline
\end{tabular}


identificó significancia estadística en el lado izquierdo de hombres en las siguientes variables: altura del lado derecho del cóndilo ( $\mathrm{p} \leq 0.0282$ ), densidad del ancho del lado medio ( $\mathrm{p} \leq 0.0312)$; en el lado derecho de hombres: densidad del ancho del lado vestibular $(\mathrm{p} \leq 0.0041)$, medio $(\mathrm{p} \leq 0.0013)$ y palatino ( $\mathrm{p} \leq 0.0452)$. En mujeres en el lado izquierdo; altura del lado derecho del cóndilo $(\mathrm{p} \leq 0.0003)$ e izquierdo $(\mathrm{p} \leq$ $0.0010)$, ancho vestibulolingual del lado derecho $(\mathrm{p} \leq 0.0091)$ e izquierdo ( $\mathrm{p} \leq 0.0257)$; en el lado derecho de mujeres: altura del lado derecho del cóndilo $(\mathrm{p} \leq 0.0004)$ e izquierdo

Tabla IV. Estadística descriptiva del cóndilo mandibular derecho de mujeres en 50 tomografías computarizadas de haz cónico.

\begin{tabular}{|c|c|c|c|c|c|c|c|c|}
\hline $\begin{array}{l}\text { Forma del } \\
\text { cóndilo }\end{array}$ & Variable & $\begin{array}{l}\text { Lado del } \\
\text { cóndilo }\end{array}$ & Media & $\begin{array}{c}\text { Error de la } \\
\text { media }\end{array}$ & $\begin{array}{l}\text { Desviación } \\
\text { estándar }\end{array}$ & Mínimo & Mediana & Máximo \\
\hline \multirow[t]{16}{*}{ Aplanada } & Altura & Derecho & 16.879 & 0.658 & 2.079 & 13.74 & 16.775 & 21.2 \\
\hline & & Izquierdo & 15.953 & 0.664 & 2.099 & 13.11 & 15.99 & 19.22 \\
\hline & $\begin{array}{l}\text { Ancho } \\
\text { vestíbulo }\end{array}$ & Derecho & 14.206 & 0.804 & 2.544 & 10.14 & 14.215 & 18.72 \\
\hline & lingual & & & & & & & \\
\hline & & Izquierdo & 14.451 & 0.728 & 2.303 & 11 & 14.31 & 17.88 \\
\hline & $\begin{array}{l}\text { Ancho frontal } \\
\text { posterior }\end{array}$ & Derecho & 7.118 & 0.399 & 1.262 & 5.06 & 7.34 & 9.09 \\
\hline & & Izquierdo & 7.181 & 0.436 & 1.377 & 4.98 & 7.31 & 8.98 \\
\hline & $\begin{array}{l}\text { Densidad de la } \\
\text { atura }\end{array}$ & Apical & 746 & 149 & 471 & 152 & 799 & 1549 \\
\hline & & Media & 398.5 & 55.5 & 175.6 & 90 & 381 & 669 \\
\hline & & Coronal & 546.6 & 70.8 & 224 & 264 & 602 & 1004 \\
\hline & $\begin{array}{l}\text { Densidad del } \\
\text { ancho }\end{array}$ & Vestibular & 567 & 78.9 & 249.5 & 267 & 548 & 1073 \\
\hline & & Media & 345.9 & 38.009 & 122.9 & 185 & 358 & 495 \\
\hline & & Palatino & 543.5 & 70.5 & 222.9 & 169 & 544 & 855 \\
\hline & Densidad pared & Anterior & 1.805 & 0.144 & 0.457 & 1.14 & 1.775 & 2.76 \\
\hline & & Posterior & 2.394 & 0.278 & 0.879 & 1.62 & 1.945 & 4.2 \\
\hline & & Superior & 2.575 & 0.317 & 1.002 & 1.62 & 2.4 & 5.17 \\
\hline \multirow[t]{15}{*}{ Ovalada } & Altura & Derecho & 17.497 & 0.733 & 2.318 & 14.47 & 17.56 & 21.91 \\
\hline & & Izquierdo & 17.292 & 0.693 & 2.193 & 14.91 & 16.935 & 20.84 \\
\hline & $\begin{array}{l}\text { Ancho } \\
\text { vestíbulo } \\
\text { lingual }\end{array}$ & Derecho & 14.082 & 0.792 & 2.503 & 10.65 & 14.665 & 18.39 \\
\hline & & Izquierdo & 12.917 & 0.828 & 2.617 & 9.8 & 11.83 & 16.71 \\
\hline & $\begin{array}{l}\text { Ancho frontal } \\
\text { posterior }\end{array}$ & Derecho & 7.481 & 0.315 & 0.997 & 5.53 & 7.555 & 9.09 \\
\hline & & Izquierdo & 7.488 & 0.365 & 1.156 & 5.46 & 7.56 & 9.2 \\
\hline & $\begin{array}{l}\text { Densidad de la } \\
\text { atura }\end{array}$ & Apical & 897 & 138 & 436 & 326 & 683 & 1585 \\
\hline & & Medio & 377.2 & 77 & 243.4 & 130 & 307 & 865 \\
\hline & & Coronal & 681.1 & 82.9 & 262.2 & 359 & 660 & 1137 \\
\hline & $\begin{array}{l}\text { Densidad del } \\
\text { ancho }\end{array}$ & Vestibular & 626.6 & 94.5 & 298.8 & 185 & 611 & 1026 \\
\hline & & Medio & 339.7 & 46.3 & 146.4 & 79 & 326.5 & 562 \\
\hline & & Palatino & 528.7 & 68.3 & 216.1 & 263 & 549 & 775 \\
\hline & Densidad pared & Anterior & 2.198 & 0.16 & 0.507 & 1.39 & 2.135 & 3.04 \\
\hline & & Posterior & 2.526 & 0.377 & 1.194 & 1.78 & 2.115 & 5.82 \\
\hline & & Superior & 2.751 & 0.396 & 1.252 & 1.75 & 2.65 & 6.07 \\
\hline
\end{tabular}


LÓPEZ, R. J. C.; GARCÍA, C. J. O.; SÁNCHEZ, M. W.; OLIVA, R. W.; GUTIÉRREZ, C. F. J. \& CÁRDENAS, J. M. Análisis morfológico del cóndilo mandibular por tomografía computarizada de haz cónico en relación al sexo en población mexicana. Int. J. Morphol., 39(5):1420-1428, 2021.

( $\mathrm{p} \leq 0.0022)$, ancho vestibulolingual del lado derecho $(\mathrm{p} \leq$ 0.0006) e izquierdo ( $\mathrm{p} \leq 0.0036)$, densidad de la pared ante- rior ( $\mathrm{p} \leq 0.0138)$; no así en los demás grupos de estudio (Tabla VII).

Tabla V. Comparación de las formas aplanada vs ovalada de cóndilo mandibular para cada variable entre los grupos de estudio.

\begin{tabular}{|c|c|c|c|c|c|}
\hline \multirow[b]{2}{*}{ Variable } & \multirow[b]{2}{*}{$\begin{array}{l}\text { Lado cóndilo } \\
\text { mandibular }\end{array}$} & \multicolumn{2}{|c|}{ Hombres } & \multicolumn{2}{|c|}{ Mujeres } \\
\hline & & Izquierdo & Derecho & Izquierdo & Derecho \\
\hline \multirow[t]{2}{*}{ Altura } & Derecho & $0.0008 *$ & $0.0172 *$ & 0.5964 & 0.9397 \\
\hline & Izquier do & $0.0046 *$ & 0.0887 & 0.2411 & 0.3845 \\
\hline \multirow[t]{2}{*}{$\begin{array}{l}\text { Ancho vestíbulo } \\
\text { lingual }\end{array}$} & Derecho & $0.0172 *$ & 0.1855 & 0.9097 & 0.3447 \\
\hline & Izquier do & 0.3445 & 1 & 0.1405 & 0.2057 \\
\hline \multirow{2}{*}{$\begin{array}{l}\text { Ancho frontal } \\
\text { posterior }\end{array}$} & Derecho & 0.5201 & 0.7335 & 0.5705 & 0.1506 \\
\hline & Izquier do & 0.0886 & 0.6229 & 0.7337 & 0.3847 \\
\hline \multirow[t]{3}{*}{ Densidad de la atura } & Apical & 0.5204 & 0.7913 & 0.2474 & 0.8501 \\
\hline & Media & 0.6775 & 0.1403 & 0.5205 & 0.9097 \\
\hline & Coronal & 0.3845 & $0.0376^{*}$ & 0.3075 & 0.8501 \\
\hline \multirow[t]{3}{*}{ Densidad del ancho } & Vestibular & 0.9397 & 0.2121 & 0.7913 & 0.5205 \\
\hline & Media & 0.2411 & 0.0173 & 0.9698 & $0.0121 *$ \\
\hline & Palatino & 0.5204 & 0.3845 & 0.9097 & 0.9698 \\
\hline \multirow[t]{3}{*}{ Densidad pared } & Anterior & 0.272 & 0.273 & 0.0754 & $0.0375^{*}$ \\
\hline & Posterior & 0.6493 & 0.2701 & 0.5671 & 0.6203 \\
\hline & Superior & 0.7912 & 0.3838 & 0.545 & 0.4961 \\
\hline
\end{tabular}

$* \mathrm{p}=\leq 0.05$

Tabla VI. Comparación de los lados derecho vs izquierdo en las formas del cóndilo mandibular ovalada y aplanada de hombres y mujeres para cada variable entre los grupos de estudio.

\begin{tabular}{|c|c|c|c|c|c|}
\hline \multirow[b]{2}{*}{ Variable } & \multirow[b]{2}{*}{ Lado cóndilo } & \multicolumn{2}{|c|}{ Hombres } & \multicolumn{2}{|c|}{ Mujeres } \\
\hline & & Aplanado & Ovalado & Aplanado & Ovalado \\
\hline \multirow[t]{2}{*}{ Altura } & Derecho & 0.7907 & 0.8197 & 0.6756 & 0.9698 \\
\hline & Izquierdo & 0.5437 & 0.7612 & 0.9092 & 0.9698 \\
\hline \multirow[t]{2}{*}{ Ancho vestíbulo lingual } & Derecho & 0.518 & 1 & 0.9698 & 0.4713 \\
\hline & Izquierdo & 0.4713 & 1 & 0.5696 & 0.7907 \\
\hline \multirow[t]{2}{*}{ Ancho frontal posterior } & Derecho & 0.9094 & 0.5421 & 0.5696 & 0.7614 \\
\hline & Izquierdo & 0.7322 & 0.5684 & 0.9698 & 0.733 \\
\hline \multirow[t]{3}{*}{ Densidad de la atura } & Apical & 0.5696 & 0.9394 & 0.7907 & 0.6767 \\
\hline & Media & 0.7907 & 0.4942 & 0.4713 & 0.9095 \\
\hline & Coronal & 0.9698 & 0.4942 & 0.6767 & 0.5696 \\
\hline \multirow[t]{3}{*}{ Densidad del ancho } & Vestibular & 0.6221 & 0.4942 & 0.426 & 0.9095 \\
\hline & Media & 0.426 & $0.0403 *$ & 0.426 & 0.4713 \\
\hline & Palatino & 0.9698 & 0.5949 & 0.733 & 0.5696 \\
\hline \multirow[t]{3}{*}{ Densidad pared } & Anterior & 1 & 1 & 0.9698 & 0.6221 \\
\hline & Posterior & 1 & 0.7314 & 0.9066 & 0.8189 \\
\hline & Superior & 0.4246 & 0.7612 & 0.8497 & 0.8793 \\
\hline
\end{tabular}

$* \mathrm{p}=\leq 0.05$

\section{DISCUSIÓN}

Hoy en día, los avances tecnológicos ofrecen una amplia lista de posibilidades en métodos diagnósticos para identificar diferencias morfológicas del complejo craneofacial. La tomografía computarizada de haz cónico ha demostrado ser el método más eficaz para analizar estructuras óseas debido a la baja dosis de radiación a la que se somete el paciente.
Zhang et al. (2016) compararon casos que presentaban sintomatología articular con tomografía computarizada de haz cónico, demostrando que existían diferencias entre ambos lados en los espacios articulares superior y anterior. En nuestro estudio no se identificaron diferencias significativas en el espacio anterior en hombres y mujeres, no así en el espacio posterior; esto puede estar relacionado con el tipo de población en la que se realizó el estudio ya que en población mexicana probablemente puedan influir las diferencias 
LÓPEZ, R. J. C.; GARCÍA, C. J. O.; SÁNCHEZ, M. W.; OLIVA, R. W.; GUTIÉRREZ, C. F. J. \& CÁRDENAS, J. M. Análisis morfológico del cóndilo mandibular por tomografía computarizada de haz cónico en relación al sexo en población mexicana. Int. J. Morphol., 39(5):1420-1428, 2021.

Tabla VII. Comparación de la forma del cóndilo mandibular derecho e izquierdo de hombres y mujeres para cada variable de estudio.

\begin{tabular}{lccccc}
\hline & & \multicolumn{3}{c}{ Hombres vs mujeres } \\
& & \multicolumn{2}{c}{ Izquierdo } & \multicolumn{2}{c}{ Derecho } \\
Variable & Lado cóndilo mandibular & Aplanado & Ovalado & Aplanado & Ovalado \\
\hline Altura & Derecho & $0.0282^{*}$ & 0.1983 & $0.0003^{*}$ & $0.0004^{*}$ \\
& Izquierdo & 0.0596 & 0.0887 & $0.0010^{*}$ & $0.0022^{*}$ \\
Ancho vestíbulo lingual & Derecho & 0.623 & 0.2565 & $0.0091^{*}$ & $0.0006^{*}$ \\
& Izquierdo & 0.5205 & 0.5205 & $0.0257^{*}$ & $0.0036^{*}$ \\
Ancho frontal posterior & Derecho & 0.9397 & 0.4495 & 0.4958 & 0.1208 \\
& Izquierdo & 0.3442 & 0.5703 & 0.8202 & 0.2565 \\
Densidad de la atura & Apical & 1 & 0.273 & 0.2411 & 0.623 \\
& Media & 1 & 0.1659 & 0.623 & 0.9097 \\
Densidad del ancho & Coronal & 0.273 & 0.1041 & 0.1403 & 0.623 \\
& Vestibular & 0.0695 & $0.0041^{*}$ & 0.0756 & 0.3845 \\
Densidad pared & Media & $0.0312^{*}$ & $0.0013^{*}$ & 0.5706 & 0.273 \\
& Palatino & 0.2123 & $0.0452^{*}$ & 0.4053 & 0.2729 \\
& Anterior & 0.1986 & 0.2727 & 0.2256 & $0.0138^{*}$ \\
& Posterior & 1 & 0.9696 & 0.4482 & 0.1394 \\
& Superior & 0.104 & 0.1211 & 0.4046 & 0.1615 \\
\hline
\end{tabular}

$* \mathrm{p}=\leq 0.05$

genéticas en el tipo de musculatura responsable del proceso de masticación.

Zhang et al. (2017) obtuvieron diferencias significativas, en ambos sexos, en el ángulo formado entre la pared posterior del cóndilo mandibular con la pared posterior de la fosa articular mediante el análisis de CBCT . Nuestro estudio abarcó un rango más amplio de edad (18 a 35 años) el cual demuestra que la morfología de la ATM se relaciona con la clase esqueletal y el tipo de musculatura. También se identificó que al ampliar el rango de edad aumenta la posibilidad de presentar mayores diferencias morfológicas en la ATM tal vez debido a un proceso adaptativo o a causas sistémicas que interfieren directamente en la articulación.

Imanimoghaddam et al. (2016) mencionaron que pacientes que padecían trastornos temporomandibulares (hombres y mujeres de 29 años) no presentaban diferencias en la morfología del cóndilo mandibular, no así en los espacios articulares. Nuestros resultados difieren de estos autores al obtener diferencias entre hombres y mujeres en la morfología condilar. Los rangos de edades son similares por lo cual se puede inferir que la edad no se relaciona directamente con la morfología del cóndilo mandibular al terminar el crecimiento y desarrollo del individuo. Las diferencias óseas del complejo dentomaxilar pueden afectar las funciones musculares responsables de la masticación por lo cual estos factores producen cambios morfológicos en la forma del cóndilo mandibular tal vez debido a la fuerza excesiva de los músculos.

Palombella et al. (2019) midieron el ancho y altura del cóndilo mandibular mediante tomografías y observaron diferencias en el primer criterio, no así en el segundo. Concordamos con los resultados obtenidos en el ancho y se identificaron diferencias en la altura del cóndilo mandibular. Estos hallazgos se pueden relacionar con la edad y en cómo esta se puede ver afectada por la pérdida de dientes, el daño a la dimensión vertical y las patologías del complejo articular.

Ishwarkumar et al. (2016) midieron la morfología del cóndilo mandibular en osamentas de acuerdo con su forma, y observaron diferencias entre hombres y mujeres, entre las cuales se destaca la forma ovalada con mayor predominancia en hombres y la forma redonda en mujeres. No comprobamos lo descrito por estos autores ya que en la población mexicana existe, predominantemente, el tipo de morfología condilar aplanada en hombres y ovalada en mujeres, tal vez debido a las características cronológicas, genéticas, étnicas y hábitos sociales que pueden influir en la morfología condilar.

Endo et al. (2011), señalaron que la posición y la morfología de la ATM analizada con CBTC en mujeres con asimetrías faciales y grupos controles mostraban mayor dimensión en los espacios articulares. Nuestros resultados no concuerdan con los de estos autores, ya que la forma oval se observa principalmente en mujeres, en la cual el ancho del cóndilo mandibular es de forma ovalada y de menores dimensiones. Con lo anterior se podría establecer una relación de sujetos clase III esqueletales en la población asiática respecto a la posición articular disminuyendo la altura condilar debido a que se encuentra desplazado mientras que en la población mexicana predomina la clase II esqueletal.

CONCLUSIÓN. La tomografía computarizada de haz cóni- 
co es un método económico, accesible y que mediante la exposición a bajas dosis de radiación, obtiene imágenes de alta resolución de los tejidos duros de la ATM, la cual permite la detección de cambios morfológicos que no pueden ser bservados en radiográficas convencionales. Se identificaron diferencias en la altura del cóndilo mandibular, probablemente debido a que la población mexicana presenta diferentes patrones esqueletales. El tipo de morfología condilar observada en el presente estudio se relaciona con el crecimiento y desarrollo. Se asume que los hombres al presentar una musculatura más fuerte propia del sexo influye directamente en los cambios morfológicos que pueden afectar el complejo de la articulación temporomandibular. El tipo esqueletal y la fuerza de la masticación se adaptan al cóndilo mandibular y factores como la raza, sexo y hábitos sociales influyen en el desarrollo de la morfología condilar.

LÓPEZ, R. J. C.; GARCÍA, C. J. O.; SÁNCHEZ, M. W.; OLIVA, R. W.; GUTIÉRREZ, C. F. J. \& CÁRDENAS, J. M. Morphological analysis of the mandibular condyle by cone beam computed tomography in relation to sex in a mexican population. Int. J. Morphol., 39(5):1420-1428, 2021.

SUMMARY: The temporomandibular joint is a double and symmetrical structure, whit a complex range of movements. Condylar morphology can vary depending on sex, age, among other factors. 100 cone beam computed tomography scans (50 men and 50 women) divided into 4 groups were analyzed. Significant differences were identified between the flattened vs oval forms on the condyle and, in turn a difference was found between the right vs left sides between men and women. Cone beam computed tomography analysis has acceptable accuracy for the diagnosis of TMJ bone abnormalities, articular components, cortical bone integrity and subcortical bone abnormalities.

KEY WORDS: Temporomandibular joint; Cone beam computed tomography; Temporomandibular disorders.

\section{REFERENCIAS BIBLIOGRÁFICAS}

Barghan, S.; Tetradis, S. \& Mallya, S. M. Application of cone beam computed tomography for assessment of the temporomandibular joints. Aust. Dent. J., 57(1):109-18, 2012.

Chavarro, P. A.; Girón, E.; Tatis, Fernando. D. \& Wilches-Luna, E. C. Evaluación de la movilidad de la articulación temporomandibular en pacientes postintubación orotraqueal mayor de 24 horas. Acta Colomb. de Cuid. Intensivo., 16(4):300-6, 2016.

Dos Anjos Pontual, M. L.; Freire, J. S. L.; Barbosa, J. M. N.; Frazão, M. A. G.; Dos Anjos Pontual, A. \& Fonseca Da Silveira M. M. Evaluation of bone changes in the temporomandibular joint using cone beam $C T$. Dentomaxillofac. Radiol., 41(1):24-29, 2012.

Endo, M.; Terajima, M.; Goto, T. K.; Tokumori, K., \& Takahashi, I.Three-dimensional analysis of the temporomandibular joint and fossa-condyle relationship. Orthodontics (Chic.)., 12(3):210-21, 2011.

Farfan, C.; Quidel, B. \& Fuentes, R. Características Anatómicas-Funcionales que Orientan la Posición del Cóndilo en la Fosa Mandibular en una. Int. J. Morphol., 38(5):1281-7, 2020.
Honey, O. B.; Scarfe, W. C.; Hilgers, M. J.; Klueber, K.; Silveira, A. M.; Haskell, B. S. \& Farman, A. G. Accuracy of cone-beam computed tomography imaging of the temporomandibular joint: Comparisons with panoramic radiology and linear tomography. Am. J. Orthod. Dentofacial Orthop., 132(4):429-38, 2007.

Imanimoghaddam, M.; Madani, A. S.; Mahdavi, P.; Bagherpour, A.; Darijani, M.; \& Ebrahimnejad, H. Evaluation of condylar positions in patients with temporomandibular disorders: A cone-beam computed tomographic study. Imaging Sci. Dent., 46(2):127-31, 2016.

Ishwarkumar, S.; Pillay, P.; Degama, B. Z. \& Satyapal, K. S. An Osteometric Evaluation of the Mandibular Condyle in a Black KwaZulu-Natal Population. Int. J. Morphol., 34(3):848-53, 2016.

Iskanderani, D.; Nilsson, M.; Alstergren, P. \& Hellén-Halme, K. Dose distributions in adult and child head phantoms for panoramic and cone beam computed tomography imaging of the temporomandibular joint. Oral Surg. Oral Med. Oral Pathol. Oral Radiol., 130(2):200-8, 2020.

Marques, A. P.; Perrella, A.; Arita, E. S.; Pereira, M. F. S. de M. \& Cavalcanti, M. de G. P. Assessment of simulated mandibular condyle bone lesions by cone beam computed tomography. Braz. Oral Res.,24(4):467-74, 2010.

Matsumoto, K.; Kameoka, S.; Amemiya, T.; Yamada, H.; Araki, M.; Iwai, K.; Hashimoto, K. \& Honda, K. Discrepancy of coronal morphology between mandibular condyle and fossa is related to pathogenesis of anterior disk displacement of the temporomandibular joint. Oral Surg. Oral Med. Oral Pathol. Oral Radiol., 116(5):626-32, 2013.

Omami, G. \& Lurie, A. Magnetic resonance imaging evaluation of discal attachment of superior head of lateral pterygoid muscle in individuals with symptomatic temporomandibular joint. Oral Surg. Oral Med. Oral Pathol. Oral Radiol., 114(5):650-57, 2012.

Palombella, A. S.; Wilson, T. D.; Fernandes, A. C. S. \& Galil, K. A. Threedimensional micro CT derived morphology of the condylus mandibulae in older adults: Implications for clinical dentistry. Int J Morphol., 37(3):78791, 2019.

Ribeiro, E. C.; Sanches, M. L.; Alonso, L. \& Smith, R. L. Shape and symmetry of human condyle and mandibular fossa shape and symmetry of human condyle and mandibular fossa. Int. J. Odontostomat, 9(1):65-72, 2015

Ros, M. L. H.; Cañete. C. E. \& Velilla M. [Magnetic resonance imaging of the temporomandibular joint]. Radiología, 50(5):377-85, 2008.

Torsten, Ö.; Carlsson G, E.; Fajers, C, M. The temporomandibular joint a morphologic study on a human autompsy material. Acta Odontol. Scand., 29(3):349-84, 1971.

Zhang, Y. L.; Song, J. L.; Xu, X. C.; Zheng, L. L.; Wang, Q. Y., Fan; Y. B. \& Liu, Z. Morphologic analysis of the temporomandibular joint between patients with facial asymmetry and asymptomatic subjects by 2D and 3D evaluation: A preliminary study. Medicine (Baltimore), 95(13):e3052, 2016.

Zhang, Y.; Xu, X. \& Liu, Z. Comparison of morphologic parameters of temporomandibular joint for asymptomatic subjects using the two-dimensional and three-dimensional measuring methods. J. Healthc. Eng., 2017(2):1-8, 2017.

Dirección para correspondencia:

Jairo Mariel Cárdenas y Francisco Javier Gutiérrez Cantú

Maestría en Ciencias Odontológicas

Facultad de Estomatología

Universidad Autónoma de San Luis Potosí

Av. Dr. Manuel Nava \# 2

Zona Universitaria C.P. 78290

San Luis Potosi S.L.P

MÉXICO

E-mail: Ilairo@hotmail.com / llairo@yahoo.com

Received: 14-06-2021

Accepted: 31-07-2021 